Círculo de Lingüística Aplicada a la Comunicación ISSN: $1576-4737$

\title{
Bremen 2019: BreMM19. Multimodality - Empirical Inroads
}

https://www.mm2014.uni-bremen.de/

September 25-27, 2019

University of Bremen, Germany

The Bremen Conferences on Multimodality are annual events with speakers from all over the world discussing and presenting recent topics of multimodal research. As an interdisciplinary and international symposium, each conference offers a place to think about developments and advancements in the topic of multimodality and the disciplines connected to this field of research. BreMM14 was dedicated to building bridges between various multimodality-ready disciplines, BreMM15 concerned itself with theoretical and methodological exploration. BreMM17 laid the foundation for the formation of a standalone discipline to be dubbed 'multimodality' as opposed to the widespread interdisciplinary view. BreMM19 will focus on empirical inroads in multimodality research.

Call Deadline: 05-Dec-2018

\section{Meeting Description}

We are happy to announce that BreMM19, the 4th Bremen Conference on Multimodality, will be held at Bremen University from September 25-27, 2019. The upcoming 4th Bremen Conference on Multimodality, BreMM19, puts empirical inroads into multimodal research centre stage. That is, it focuses on the study of multimodal artefacts and performances through investigating (larger collections of) actual data. It seeks to further the empirical validation and evaluation of both widely established and new hypotheses and theories about multimodal meaning-making. Such novel approaches have been in the focus of vivid discussions and have often become complex subjects of inquiry in their own right. 


\section{Call for Papers}

Please see website for full version of this Call for Papers. As with previous BreMM conferences, we view BreMM19 as an essentially interdisciplinary endeavor. Therefore, we call for papers from scholars of all disciplines doing empirical analyses of multimodal artefacts and performances. We particularly invite cooperative research projects that involve several disciplines and expertise from various fields, in search of novel mixed-method approaches.

Proposals may address the following questions:

- What are the relevant practices and latest tools for multimodal corpus design?

- What will be the particular challenges for empirical work raised when we consider an ever broader range of multimodal phenomena?

- How are multimodal corpora then to be conceptualized, and how do various definitions of 'mode' affect corpus design? What are multimodal corpora?

- How can new corpus methods and research practices be tailored to support multimodal work?

- What are the most common disciplinary sources and theoretical starting points for large-scale, corpus-based multimodal research projects?

- How may empirical multimodal research intersect with or strengthen endeavors in the humanities and social sciences? What are the complementarities, limits, and opportunities?

- Can multimodal corpus research be acquired in disciplines not traditionally aligned with it? What examples are there of successful applications of corpus methods in non-traditional academic environments (e.g., design, marketing, advertising, investigative journalism)?

- How can we make multimodal methods accessible and usable to the wider academic and professional public? How do we create multimodal awareness with empirical methods?

\section{Submission}

Please submit your proposal as an e-mail attachment (Word, PDF) by December 5, 2018, to bremm19uni-bremen.de.

For further information, visit the conference website: http://unihb.eu/BreMM19 Notification of acceptance by end of December 2018. 